\title{
Suppression of Superfluidity upon Overflow of Trapped Fermions: Quantal and Thomas-Fermi Studies
}

\author{
P. Schuck ${ }^{1,2}$ and X. Viñas ${ }^{3}$ \\ ${ }^{1}$ Institut de Physique Nucléaire, IN2P3-CNRS, Université Paris-Sud, F-91406 Orsay-Cédex, France \\ ${ }^{2}$ Laboratoire de Physique et Modélisation des Milieux Condensés, CNRS and Université Joseph Fourier, \\ 25 Avenue des Martyrs, Boîte Postale 166, F-38042 Grenoble Cedex 9, France \\ ${ }^{3}$ Departament d'Estructura i Constituents de la Matèria and Institut de Ciències del Cosmos, Facultat de Física, \\ Universitat de Barcelona, Diagonal 647, E-08028 Barcelona, Spain
}

(Received 15 July 2011; published 7 November 2011)

\begin{abstract}
Two issues are treated in this work. (i) The generic fact that, if a fermionic superfluid in the BCS regime overflows from a narrow container into a much wider one, pairing is much suppressed at the overflow point. Physical examples where this feature may play an important role are discussed. (ii) A ThomasFermi approach to inhomogeneous superfluid Fermi systems is presented and shown to work well in cases where the local density approximation breaks down.
\end{abstract}

DOI: 10.1103/PhysRevLett.107.205301

PACS numbers: 67.60. $-\mathrm{g}$, 05.30.Fk

Superfluid fermions in finite systems can exist in traps of cold atoms, in nuclear systems, in small metallic clusters, etc. An interesting question arises as to what happens to the superfluid if its Fermi level reaches the edge of a finite container; i.e., either the fluid overflows into the continuum or it pours into another container of much larger dimension. A trapping potential of this type has already been generated experimentally for the study of cold bosonic atoms [1]. It should also be possible to use it for fermionic atoms [2].

In the inner crust of neutron stars, there also may occur the situation where a superfluid neutron gas of variable density coexists in between the lattice of (superfluid) nuclei $[3,4]$. This situation often is mimicked by a WignerSeitz cell, where the single-particle potential has a pocket, representing the nucleus, embedded in a large container. Still other systems may exist with similar situations.

The purpose of this work is to study superfluidity of fermions at the overflow (drip) in the BCS regime. Since the quantal solution of BCS equations in geometries with rapidly varying single-particle potentials with a large number of particles is numerically difficult, we will present, as a second objective of this work, a Thomas-Fermi (TF) approach to inhomogeneous superfluidity which shows good performance in situations where local density approximation (LDA) fails.

For our study, we first will use a schematic model of slab geometry with a transverse potential of large extension $L$ possessing at the origin a "pocket" of variable depth and size $R$ much smaller than the outer container. Schematically, such a potential is shown in Fig. 1(a). This slab configuration may roughly mock up one sheet of a so-called Lasagne configuration in the inner crust of neutron stars [5]. We, therefore, will use nuclear dimensions for the slab model, but they can easily be replaced by dimensions relevant for other systems. Our model and the ensuing generalizations treated below, therefore, are believed to be generic. We will study the slab configuration also because the quantal solution of the gap equation is evaluated relatively directly and the quality of the TF approach can thus be established. Once this is achieved, we will also go over to other geometrical configurations. We, for instance, will treat a second potential shown in Fig. 1(b) with spherical symmetry, a kind of which, as already mentioned, has been used for bosonic atoms in [1].

The wave functions and eigenenergies of a box, as shown in Fig. 1(a) with a potential hole, are given in [6]. For pairing, we use a contact force with a cutoff $\Lambda$, to make things simple. Integrating over momenta in the slab direction the usual gap equation $\Delta_{n}=\sum_{n^{\prime}} \int \frac{d^{2} p}{(2 \pi \hbar)^{2}} V_{n n^{\prime}} \Theta\left(\Lambda-\varepsilon_{n^{\prime}}-\varepsilon_{p}\right) \Delta_{n^{\prime}} /\left[2 E_{n^{\prime}}(p)\right]$ with $E_{n}(p)=\sqrt{\left(\varepsilon_{n}+\varepsilon_{p}-\mu\right)^{2}+\Delta_{n}^{2}}$ the quasiparticle energy, $\Theta(x)$ the step function, and $\varepsilon_{n}$ and $\varepsilon_{p}$ the discrete single-particle energies in the transverse direction and the kinetic energies in the slab direction, respectively-one arrives at the following gap equation: (a)

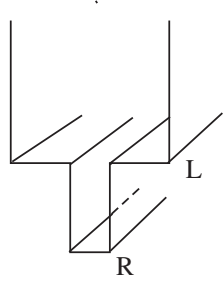

(b)

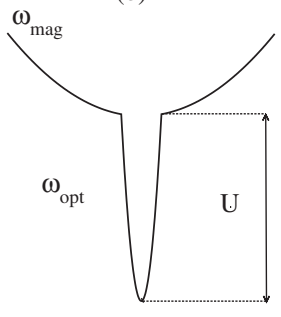

FIG. 1. Schematic view of the potentials used in this work. Panel (a) shows a perspective view of the slab potential which is translationally invariant parallel to the slab direction. Panel (b) represents a spherically symmetric container. 


$$
\Delta_{n}=-\sum_{n^{\prime}} \Theta\left(\Lambda-\varepsilon_{n^{\prime}}\right) V_{n n^{\prime}} K_{n^{\prime}},
$$

with

$$
K_{n}=\frac{m}{4 \pi \hbar^{2}} \Delta_{n} \ln \frac{\Lambda-\mu+\sqrt{(\Lambda-\mu)^{2}+\Delta_{n}^{2}}}{\varepsilon_{n}-\mu+\sqrt{\left(\varepsilon_{n}-\mu\right)^{2}+\Delta_{n}^{2}}},
$$

where $m$ is the particle mass and the indices $n$ stand for the level quantum numbers in the confining potential of Fig. 1(a). The matrix elements $V_{n n^{\prime}}=$ $-g \int_{-L}^{+L}\left|\varphi_{n}(z)\right|^{2}\left|\varphi_{n^{\prime}}(z)\right|^{2} d z$ of the contact force $-g \delta(\mathbf{r}-$ $\left.\mathbf{r}^{\prime}\right)$ can be evaluated straightforwardly from the wave functions $\varphi_{n}(z)$ given in [6].

For an example, we take as a cutoff $\Lambda=50 \mathrm{MeV}$ counted from the edge of the pocket potential whose depth is $V_{0}=-40 \mathrm{MeV}$. Its extension ranges from $-R$ to $+R$, with $R=10 \mathrm{fm}$. The wide potential with infinite walls has an extension from $-L$ to $+L$, with $L=100 \mathrm{fm}$. For the coupling strength, we take $g=150 \mathrm{MeV} \mathrm{fm}^{3}$.

Before we show the results, let us explain our TF approach for this problem. For this, we transform Eq. (1) into a continuum version in the following way. We first consider the Wigner transform of the density matrix corresponding to the state $|n\rangle-\left[\hat{\rho}_{n}\right]_{W}=[|n\rangle\langle n|]_{W}$-and take the $\hbar \rightarrow 0$ limit of this expression (see [7-9]):

$$
f_{E}(z, p)=\frac{1}{g^{\mathrm{TF}}(E)} \delta\left(E-H_{\mathrm{cl}}\right)+O\left(\hbar^{2}\right),
$$

where $H_{\mathrm{cl}}=\frac{p^{2}}{2 m}+V(z)$ is the classical Hamiltonian, with $V(z)$ the potential [Fig. 1(a)] in the transverse direction and $g^{\mathrm{TF}}(E)$ the corresponding level density to the lowest order in $\hbar$, i.e., the usual TF expression [9]. Quantum numbers and energies are simply characterized by the continuous energy variable $E$, which takes the place of the discrete values $\varepsilon_{n}$ in the quantal case.

The TF version of the gap equation (1) then reads

$$
\Delta(E)=-\int_{V_{0}}^{\Lambda} d E^{\prime} g\left(E^{\prime}\right) V\left(E, E^{\prime}\right) K\left(E^{\prime}\right),
$$

with $K(E)$ an obvious generalization of $K_{n}$. The matrix elements $V\left(E, E^{\prime}\right)$ can be evaluated in replacing $\left|\varphi_{n}(z)\right|^{2}$ by [7] $\rho_{E}^{\mathrm{TF}}(z)=\frac{1}{g^{\mathrm{TF}}(E)} \frac{1}{2 \pi}\left(\frac{2 m}{\hbar^{2}}\right)^{1 / 2}[E-V(z)]^{-1 / 2}$, the on-shell TF density in the transverse direction. As the reader will easily realize, the way of proceeding is very different from usual LDA, where the finite-size dependence is put into the (local) chemical potential, whereas here it is put into the matrix elements of the pairing force.

We are now in a position to solve the quantal and TF gap equations for the abovementioned parameter values of our model. The result for the gap at the chemical potential $\mu$ is shown in Fig. 2 as a function of $\mu$. We start with $\mu$ from the bottom of the pocket well, i.e., with zero density. We then increase $\mu$, i.e., the density. We see that, once the fillup of the pocket reaches its top, the values of the gap sharply

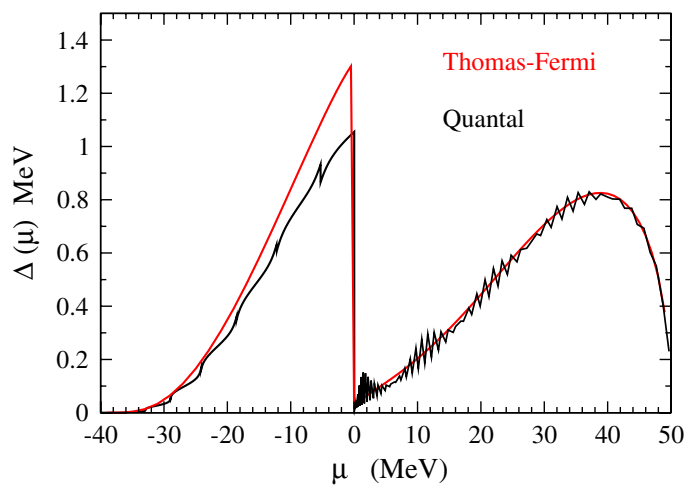

FIG. 2 (color online). Quantal and TF pairing gap in the slab geometry as a function of the chemical potential.

drop and practically reach zero. In the continuum, the gaps slowly rise again. We see that quantal and TF values are in close agreement. The overshoot of the TF solution for negative $\mu$ is very likely due to the smallness of the pocket, which only can accommodate nine bound levels. It may be partially cured including $\hbar$ corrections [9], which, however, we do not consider here. Before we come to an explanation of the drop of the gaps at overflow (drip), let us study the gaps as a function of position in the transverse direction: $\Delta(z)=-g K(z)$, with $K(z)=\sum K_{n}\left|\varphi_{n}(z)\right|^{2}$. Semiclassically, this expression becomes $K(z)=$ $\int_{V_{0}}^{\Lambda} d E g^{\mathrm{TF}}(E) K(E) \rho_{E}^{\mathrm{TF}}(z)$.

In Fig. 3, we show the density profiles for three values of $\mu: \mu=40,0.5$, and $-5 \mathrm{MeV}$. We see that quantal and TF results agree, up to shell fluctuations, very well. We also show the LDA results. We see that they can be as wrong as by $50 \%$. For other choices of system parameters, the LDA error may even be worse. This stems from the fact that, in TF (and, of course, also quantally), there is coupling between inside and outside the pocket; i.e., the Cooper pair wave function extends into both regions. This tends to equilibrate the values of the gaps. In LDA, the contrast is

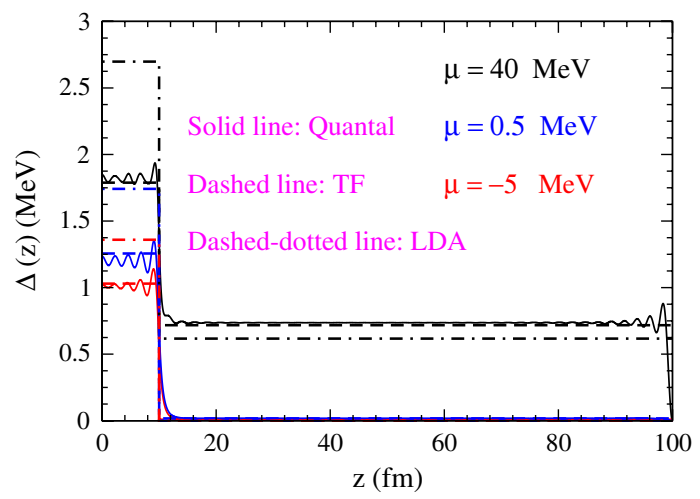

FIG. 3 (color online). Position dependence of the gap in the slab geometry for different values of the chemical potential. Quantal, TF, and LDA results are shown. Notice that $\Delta$ for $\mu=0.5$ and $-5.0 \mathrm{MeV}$ is practically zero in the gas region. 
much too strong. The drop of the gaps when crossing the threshold can be explained by the fact that the singleparticle states are strongly delocalized in the outer container, and, thus, their contribution to the pairing matrix element $V_{n, n^{\prime}}$ becomes very small.

Having gained faith in our TF approach, we now can explore other geometries and other systems, which are more difficult for quantal solutions. In Fig. 4, we show the result for $\Delta$ in the spherical double harmonic oscillator potential shown in Fig. 1(b) which may be realized with cold fermionic atoms to study the overflow situation. A zero range force with strength $g=-1.0$ and cutoff $\Lambda=$ 164.34 (in the corresponding optical trap units with $\omega_{\text {opt }}=$ $2 \pi \times 1000 \mathrm{~Hz}$ taken from [2]) is used. We see that the result is qualitatively similar to the slab case, although, in this spherical geometry, the dip does not quite reach zero and also is shifted slightly to an energy above the break of the potential. Note that this depends strongly on the choice of the ratio $\omega_{\mathrm{mag}} / \omega_{\mathrm{opt}}$ as it can be seen in Fig. 4. Also, the gap starts to decrease towards the minimum quite early. It shall be interesting to see whether our prediction can be verified experimentally.

Let us now make a more realistic study of Wigner-Seitz (WS) cells including electrons in $\beta$ equilibrium to simulate the inner crust of neutron stars [5]. To this end, the mean field is computed self-consistently using the BarcelonaCatania-Paris (BCP) energy density functional [10] together with the TF approach, as explained in [11]. The semiclassical description of the WS cells including pairing correlations at the TF level is obtained from this mean field using the finite-range part of the Gogny D1S force [12] in the pairing channel [13]. It must be pointed out that the total energy per baryon obtained with our TF approach is in very good agreement with the old quantal calculation of Negele and Vautherin [14], as it is explicitly discussed in

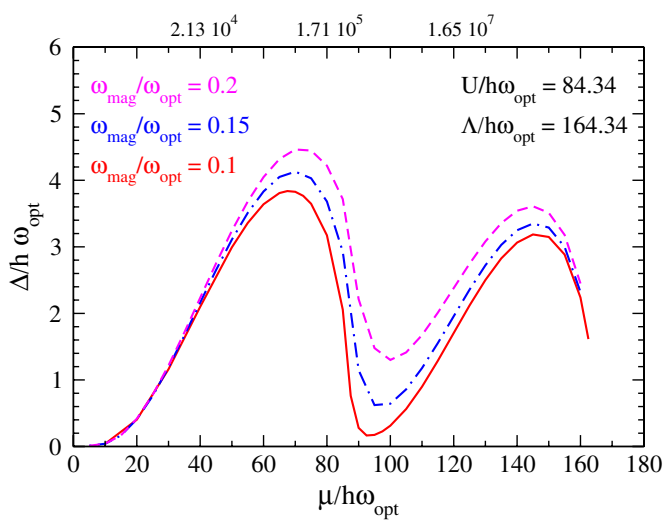

FIG. 4 (color online). Average TF gaps at the Fermi energy as a function of the chemical potential for the potential shown in Fig. 1(b). In the completely filled optical trap $(\mu=U)$, we accommodate $10^{5}$ atoms in each spin state. The total number of atoms in the trap with $\mu / \hbar \omega_{\mathrm{opt}}=40,80$, and 120 are indicated in the upper horizontal axis.
Ref. [11]. In Fig. 5 are displayed the corresponding gaps with their radius dependences. It is seen that, when the gap is small outside the region of the nucleus, then the gap also is small inside the nucleus. This stems from the very large coherence length, where one neutron of a Cooper pair can be in the huge volume of the gas and the other inside the small volume of the nucleus (proximity effect). In this way, the gas imprints its behavior for the gap also inside the nucleus. Such a conclusion was also given in a quantal Hartree-Fock-Bogoliubov (HFB) calculation by Grasso et al. in [15], which shows that the here-employed BCS approximation apparently yields very similar answers to the ones from a full HFB calculation for WS cells $[3,16]$. Finally, in the lower panel of Fig. 5, we show a comparison of LDA and present TF results for a particular WS cell. We see a huge difference in the surface region of the nucleus. This simply stems from the fact that, in the case of the ${ }_{40}^{500} \mathrm{Zr}$ nucleus in the WS cell, the gap is very small, and, therefore, the coherence length is very large, invalidating LDA. A study with examples a little less unfavorable for LDA is given in [17].

For isolated nuclei at the neutron drip, the situation may be different. It seems that, in this situation, the difference
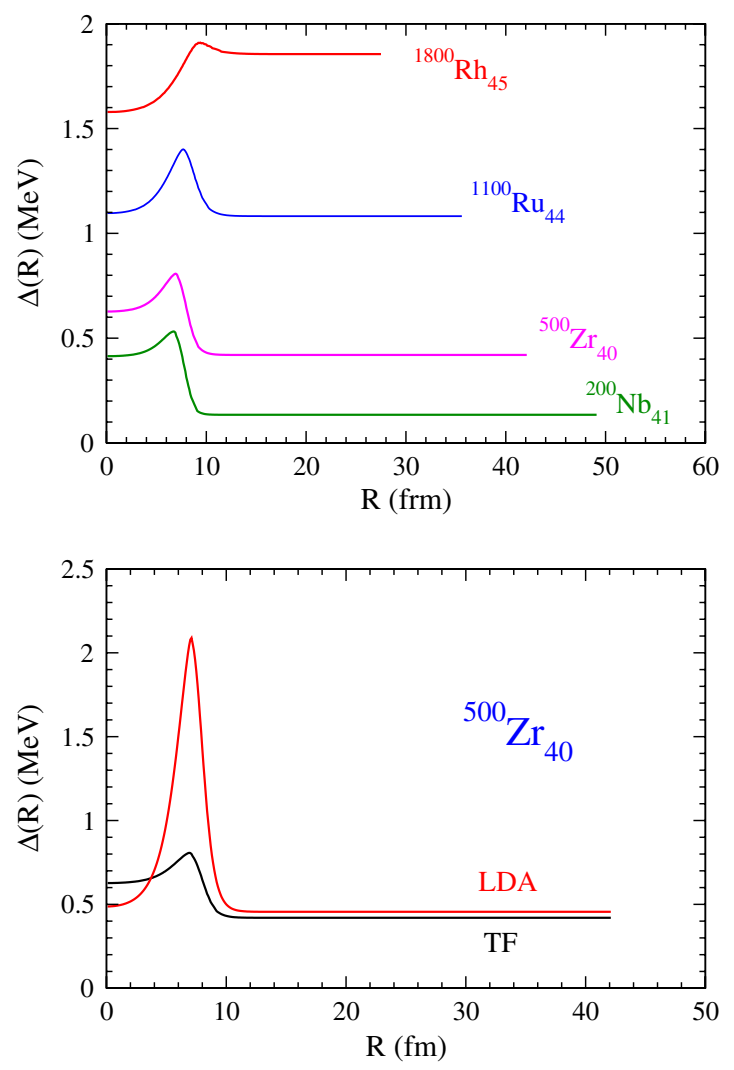

FIG. 5 (color online). Upper panel: radial dependence of the TF gap in the considered WS cells. The end points indicate the radius of the WS cells. Lower panel: comparison between TF and LDA gaps as a function of the position in a WS cell containing a single ${ }_{40}^{500} \mathrm{Zr}$ nucleus. 
between HFB and BCS approaches may be significant. Somewhat conflicting results in this respect exist in the literature. In Ref. [18], very similar results to ours are found for $S$-wave pairing. On the other hand, in [19], the gap seems to rise towards the drip before it bends down. Similar results have recently been found in [20]. Preliminary investigations show that these discrepancies may be due to large shell fluctuations in isolated nuclei. More studies in this direction seem to be necessary.

Summarizing, we have studied superfluid fermions in a large container, either external (cold atoms) or created selfconsistently (nuclei) for situations where the top of the fluid reaches the edge of a small pocket situated at the origin of the wide confining potential. The gap drops to zero at the edge before rising again when the density fills up the outer container. This at first somewhat surprising phenomenon can be explained quite straightforwardly. Such situations, as already mentioned, can exist in cold atoms and nuclei in the inner crust of neutron stars, two examples treated here with their specific forms of containers. For small systems like isolated nuclei at the neutron drip, the situation may be blurred by shell effects.

As an important second aspect of this Letter, we showed that a novel Thomas-Fermi approach to inhomogeneous situations can cope with situations where LDA fails. This means that our TF approach is free of the restrictive condition, prevailing for LDA, that the Cooper pair coherence length must be shorter than a typical length $l$ (the oscillator length in the case of a harmonic container) over which the mean field varies appreciably. On the contrary, our TF theory has the usual TF validity criterion, namely, that local wavelengths must be shorter than $l$.

The accuracy of our TF approach opens wide perspectives for a treatment of inhomogeneous superfluid Fermi systems, with a great number of particles not accessible for a quantal solution of the BCS (HFB) equations. Such systems may be cold atoms in deformed containers (eventually reaching millions of particles), superfluid-normalfluid interfaces, vortex profiles, etc. As a matter of fact, as is well-known [9], the TF approach becomes the more accurate, the larger the system. Thus, the TF approximation is complementary to the quantal one in the sense that the former works where the latter is difficult or even impossible to be obtained numerically.

We thank W. Ketterle and A. Minguzzi for their interest in this work and for pointing out Refs. [1,2]. We are grateful to Michel Farine for contributions and to Michael Urban for useful discussions and a critical reading of the manuscript. We also thank K. Hagino for pointing to
Ref. [19] and sending their own results prior to publication. This work has been partially supported by the IN2P3CAICYT Collaboration (ACI-10-000592). One of us (X. V.) acknowledges Grants No. FIS2008-01661 (Spain and FEDER) and No. 2009SGR-1289 (Spain) and the Consolider Ingenio Programme CSD2007-00042 for financial support.

[1] D. M. Stamper-Kurn, H.-J. Miesner, A. P. Chikkatur, S. Inouye, J. Stenger, and W. Ketterle, Phys. Rev. Lett. 81, 2194 (1998).

[2] L. Viverit, S. Giorgini, L. P. Pitaevskii, and S. Stringari, Phys. Rev. A 63, 033603 (2001).

[3] M. Baldo, U. Lombardo, E. E. Saperstein, and S. V. Tolokonnikov, Nucl. Phys. A750, 409 (2005); M. Baldo, E. E. Saperstein, and S. V. Tolokonnikov, Eur. Phys. J. A 32, 97 (2007).

[4] N. Chamel, S. Goriely, J. M. Pearson, and M. Onsi, Phys. Rev. C 81, 045804 (2010).

[5] P. Haensel, Neutron Stars 1: Equation of State and Structure (Springer-Verlag, New York, 2007).

[6] S. Flügge, Practical Quantum Mechanics (SpringerVerlag, Berlin, 1974).

[7] X. Viñas, P. Schuck, M. Farine, and M. Centelles, Phys. Rev. C 67, 054307 (2003).

[8] X. Viñas, P. Schuck, and M. Farine, Int. J. Mod. Phys. E 20, 399 (2011).

[9] P. Ring and P. Schuck, The Nuclear Many-Body Problem (Springer-Verlag, Berlin, 1980).

[10] M. Baldo, P. Schuck, and X. Viñas, Phys. Lett. B 663, 390 (2008).

[11] X. Viñas, P. Schuck, and M. Farine, arXiv:1106.0187; J. Phys. Conf. Ser. 321, 012024 (2011).

[12] J. Dechargé and D. Gogny, Phys. Rev. C 21, 1568 (1980); J.-F. Berger, M. Girod, and D. Gogny, Comput. Phys. Commun. 63, 365 (1991).

[13] The D1S force for pairing has been multiplied with a factor of 0.85 to compensate for the use of the bare mass in the calculation.

[14] J. W. Negele and D. Vautherin, Nucl. Phys. A207, 298 (1973).

[15] M. Grasso, E. Khan, J. Margueron, and N. Van Giai, Nucl. Phys. A807, 1 (2008).

[16] Our TF theory can be generalized to the HFB case, which shall be done in future work.

[17] A. Pastore, F. Barranco, R. A. Broglia, and E. Vigezzi, Phys. Rev. C 78, 024315 (2008).

[18] I. Hamamoto, Phys. Rev. C 71, 037302 (2005).

[19] N. Tajima, Eur. Phys. J. A 25, 571 (2005).

[20] K. Hagino and H. Sagawa, arXiv:1105.5469. 